\title{
Depression und Suizidalität
}

\author{
Ulrich Hegerl \\ Psychiatrische Klinik der Ludwig-Maximilians-Universität München, Deutschland
}

\section{Schlüsselwörter \\ Depression · Suizid · Antidepressiva · Prävention, Suizid · Gemeindebasierte Intervention}

\section{Zusammenfassung}

Auch wenn die Freiheit zur Selbsttötung zu unserer menschlichen Existenz gehört, so erfolgen zirka 90\% aller Suizide im Rahmen psychiatrischer Erkrankungen und damit in Zuständen mit eingeschränkter Urteilskraft. Depressive Erkrankungen stellen die häufigste psychiatrische Ursache für Suizide dar. Die Optimierung der Versorgung depressiver Patienten ist somit eine der aussichtsreichsten Strategien zur Suizidprävention. Im Rahmen des Nürnberger Bündnisses gegen Depression konnte durch eine Kooperation mit Hausärzten, Multiplikatoren wie Lehrern, Pfarrern, Altenpflegekräften und Medien, durch eine intensive Öffentlichkeitsarbeit sowie durch Unterstützung der Selbsthilfe eine deutlich Reduktion suizidaler Handlungen gegenüber einem als Baseline zugrunde gelegten Vergleichsjahr und der Kontrollregion Würzburg erzielt werden. Dieser Ansatz wird nun im Rahmen des Deutschen Bündnisses gegen Depression sowie des von der Europäischen Kommission geförderten Projektes "European Alliance Against Depression» (EAAD) weiter verfolgt. Im letzten Abschnitt des Beitrags wird die suizidpräventive, jedoch auch mögliche Suizid induzierende Wirkung von Antidepressiva diskutiert.

Das Wissen um die Möglichkeit, unter schlimmsten Umständen auch den Freitod wählen zu können, gehört zu unserem Menschsein. Sie ist Teil unserer Freiheit, auf die wir so stolz sind, und kann in sehr schwierigen Lebensumständen auch Tröstliches haben. Wir lassen uns deshalb die Möglichkeit

\section{Key Words}

Depression - Suicide - Antidepressants ·

Prevention, suicide $\cdot$ Community based intervention

\section{Summary}

Depression and Suicidality

Even if the freedom to suicide is part of our human existence, about $90 \%$ of all suicides occur in the context of psychiatric disorders and thus in states of limited power of judgment. Depressive disorders represent the most frequent cause for suicides. Thus, optimization of medical care for depressive patients is one of the most promising strategies to prevent suicides. In the context of the 'Nuremberg Alliance Against Depression' it came to an obvious reduction of suicidal acts compared to a baseline year and compared to the control region of Würzburg. The reduction could be reached by a cooperation with GPs, multipliers such as teachers, priests, geriatric caregivers and the media, through intensive public relations work and through support of self help activities. This approach is carried forward within the Germanywide 'Alliance Against Depression' and within the 'European Alliance Against Depression' (EAAD) which is funded by the European Commission. In the last part of the article the suicide-preventive, but also the possible suicide-inducing effect of antidepressants is discussed.

eines Freitodes nicht gerne in Abrede stellen. Tatsache ist jedoch, dass die meisten Selbsttötungen im Rahmen psychiatrischer Erkrankungen und am häufigsten im Rahmen depressiver Erkrankungen erfolgen und so aus medizinischer Sicht eher als Folge einer Erkrankung denn als freie Entscheidung

\begin{tabular}{ll}
\hline KARGER & ๑ 2005 S. Karger GmbH, Freiburg \\
Fax +49 7614520714 & Accessible online at: \\
$\begin{array}{l}\text { E-mail Information@Karger.de } \\
\text { www.karger.com }\end{array}$ & www.karger.com/ver
\end{tabular}


aufzufassen sind. In dieser Arbeit sollen drei Aspekte angesprochen werden, die sich aus der Überlappung zwischen den Phänomenen Depression und Suizidalität ergeben: 1. Epidemiologische Daten zur Überlappung von Depression und Suizidalität; 2. Suizidprävention durch Optimierung der Versorgung depressiver Patienten; 3. Effekte von Antidepressiva auf Suizidalität.

\section{Epidemiologische Daten zur Überlappung von Depression und Suizidalität}

Unter «Suizidalität» werden Gedanken, Impulse und Handlungen zusammengefasst, die auf Selbsttötung abzielen. Unter diesen Begriff fallen demnach sowohl Suizidversuche als auch Suizide. Dies sind sehr unterschiedliche Phänomene, wie sich an ihren sozioepidemiologischen Unterschieden deutlich manifestiert. Das Suizidrisiko ist für Männer höher als für Frauen und nimmt mit dem Alter zu. Ein alter Mann hat ein etwa 20-mal höheres Suizidrisiko als eine junge Frau. Genau umgekehrt ist die Situation bei Suizidversuchen. Junge Frauen haben das höchste Suizidversuchsrisiko. Aussagen zu Suiziden lassen sich demnach nicht auf Suizidversuche übertragen und umgekehrt.

Auch unter Depression werden unterschiedliche Phänomene zusammengefasst wie wahnhafte Depression, Dysthymie und depressive Episoden im Rahmen einer bipolaren affektiven Störungen. Jedes davon ist zweifelsohne in unterschiedlicher Weise mit Suizidalität verknüpft. Psychologische Autopsiestudien ergaben, dass über 90\% aller Suizidopfer unter psychiatrischen Erkrankungen litten, am häufigsten unter Depressionen, gefolgt von Alkoholabhängigkeit und Schizophrenie. Der Anteil depressiv Erkrankter an den Suizidenten lag zwischen 30 und 90\% [Lönnqvist, 2000; Cheng, 1995]. Suizide erscheinen nach diesen Studien in erster Linie als Folge psychiatrischer Erkrankungen und weniger als Folge krankheitsunabhängiger schwieriger Lebensumstände. Letzteren, abgebildet durch Parameter wie Arbeitslosigkeit oder Familienstand wird in der öffentlichen Meinung häufig ein höherer Stellenwert in diesem Zusammenhang zugesprochen. Mortensen et al. [2000] fanden in einer bevölkerungsbasierten Fallkontrollstudie (5\% der dänischen Bevölkerung), dass sich fast $50 \%$ der 811 Suizidenten in stationärer psychiatrischer Behandlung befanden oder befunden hatten. Arbeitslosigkeit, geringes Einkommen und Leben ohne Partnerschaft korrelierten ebenfalls mit einem erhöhten Suizidrisiko. In einer multivariaten Analyse erwies sich jedoch der Faktor «Psychiatrische Erkrankung mit stationärer Behandlung» als stärkster Prädiktor, während die Bedeutung der anderen Faktoren abnahm. Nach Adjustierung für Effekte durch den Faktor «Psychiatrische Erkrankung» ließen sich lediglich 3\% der Suizide auf sozioökonomische Risikofaktoren zurückführen. Diese Ergebnisse weisen darauf hin, dass Zusammenhänge zwischen Suizidrisiko und Arbeitslosigkeit oder anderen sozioökonomischen Variablen leicht überschätzt werden, da sich die Zusammenhänge auch indirekt durch negative Einflüsse der Depression auf diese Variablen ergeben können. Wird ein höheres Suizidrisiko bei Arbeitslosen gefunden, so kann dieser Zusammenhang lediglich Folge dessen sein, dass Menschen mit rezidivierenden Depressionen oder Alkoholabhängigkeit leicht in Gefahr geraten, ihre Arbeit zu verlieren. Die Autoren weisen auf die sich hieraus ergebende Konsequenz hin, dass die Optimierung der Versorgung psychisch kranker Menschen eine der vielversprechendsten Strategien zur Suizidprävention darstellt.

Bei Patienten mit schwereren, rezidivierenden Depressionen, die einer stationären Behandlung bedürfen, wurde über das ganze Leben hinweg eine Suizidmortalität zwischen 10 und $15 \%$ berechnet [Guze and Robins, 1970]. Wird auch das Gros der leichter depressiv Erkrankten und ambulant behandelten Patienten berücksichtigt, wird das Risiko, im Laufe des Lebens durch Suizid zu versterben, auf 3-6\% geschätzt, wobei dieses Risiko für Männer mit 7\% deutlich höher als für Frauen mit 1\% liegt. [Inskip et al., 1998; Blair-West et al., 1999]. Unipolare Depressionen scheinen sogar ein etwas höheres Suizidrisiko zu bergen als bipolare affektive Störungen [Angst et al., 2002; Brent et al., 1993].

Das Suizidrisiko scheint nach retrospektiven und prospektiven Studien zu Beginn der depressiven Erkrankung und zu Beginn depressiver Episoden sowie in den Wochen und Monaten nach der Entlassung am höchsten zu sein [Tsuang and Woolson, 1978; Black et al., 1989; Clayton, 1983; Angst, 1999; Mortensen et al., 2000].

\section{Suizidprävention durch Optimierung der Versorgung depressiver Patienten: vom Nürnberger Bündnis zum Europäischen Bündnis gegen Depression}

Die Versorgung depressiv Erkrankter ist optimierungsbedürftig. Obwohl mit Pharmakotherapie und Psychotherapie bewährte Behandlungsmöglichkeiten mit belegter Wirksamkeit zur Verfügung stehen, erhält derzeit nur eine Minderheit der Patienten eine optimale Therapie. Rund die Hälfte der Betroffenen sucht aus Unkenntnis, krankheitsbedingter Hoffnungslosigkeit oder Schamgefühlen heraus keine professionelle Hilfe auf [Wittchen et al., 2000]. Wissensdefizite zu Depression beschränken sich nicht nur auf Patienten, sondern zeigen sich ebenfalls in weiten Teilen der Bevölkerung [Althaus et al., 2002] und bei Berufsgruppen, die in ihrer täglichen Arbeit mit dem Phänomen Depression konfrontiert sind (z.B. Lehrer, Seelsorger, Altenpfegekräfte, Berater usw.). Für den Hausarzt wird das Erkennen einer depressiven Störungen oft dadurch erschwert, dass sich rund $70 \%$ der depressiven Patienten primär aufgrund körperlicher Beschwerden im Rahmen der Depression an den Arzt wenden [Simon et al., 1999]. Wird die Depression erkannt, so wird oft nicht konsequent behandelt, nicht mit dem richtigen Medikament, nicht in der richtigen Dosierung, nicht über einen ausreichenden Zeitraum. Hinzu 


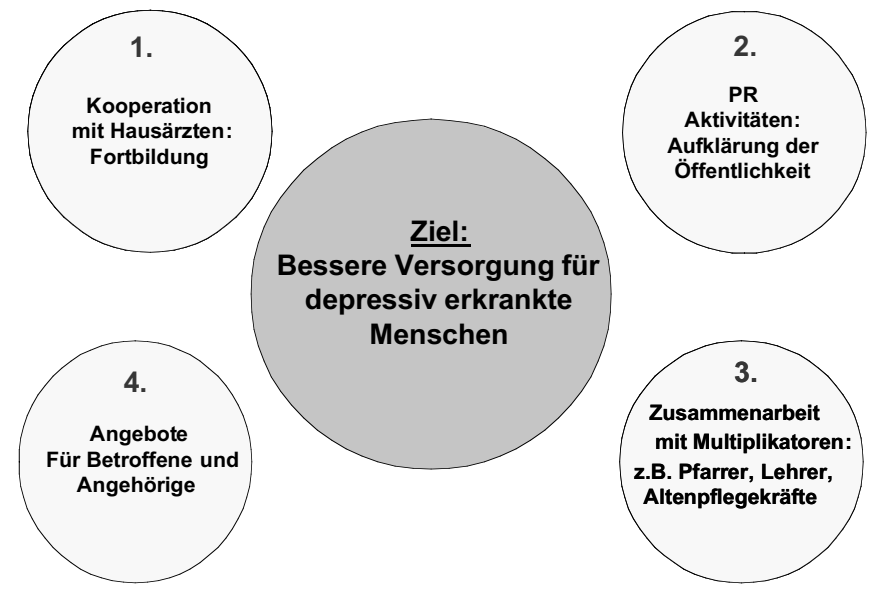

Abb. 1. Der Vier-Ebenen-Ansatz des Nürnberger Bündnisses gegen Depression zur Verbesserung der Versorgung depressiv erkrankter Menschen.

kommen dann noch Compliance-Probleme. Häufig fürchten sich Patienten vor der notwendigen längerfristigen Behandlung aus pauschalen Ängsten gegenüber Psychopharmaka («ich will nicht süchtig werden») [Hegerl et al., 2003].

Vor diesem Hintergrund wurde im Jahr 2000 das «Nürnberger Bündnis gegen Depression» als Projekt des Kompetenznetzes «Depression, Suizidalität» (gefördert vom Bundesministerium für Bildung und Forschung, BMBF, siehe www.kompetenznetz-depression.de) gestartet mit dem Ziel, die Versorgung depressiv Erkrankter zu verbessern und über diesen Weg die Suizidalität günstig zu beeinflussen. Partner im «Nürnberger Bündnis gegen Depression» sind Ärzte, Psychotherapeuten, Beratungsstellen, Kliniken, Schulen, Apotheken, Kirchen, Selbsthilfe- und Angehörigengruppen und viele weitere Einrichtungen. Die Intervention erfolgte auf folgenden vier Ebenen (Abb. 1):

1. Kooperation mit Hausärzten: In den Jahren 2001 und 2002 fanden in Nürnberg 12 Fortbildungen für Ärzte statt. Dabei wurde z.B. das Screening nach Depressionen mit Hilfe des WHO-5 [WHO, 1998; Henkel et al., 2003] empfohlen oder per Rollenspiel die Exploration und der Umgang mit Suizidalität geübt. Zur Unterstützung der Behandlung erhielten die Ärzte vielfältige Informationsmaterialien (Broschüren, Flyer, Videos) zur Weitergabe an Patienten.

2. Öffentlichkeitsarbeit: Eine professionelle Öffentlichkeitskampagne wurde entwickelt mit den Kernbotschaften «Depression kann jeden treffen», «Depression hat viele Gesichter», «Depression ist erfolgreich behandelbar». Zur Umsetzung kamen Großplakate, Poster (Abb. 2) und ein Kinospot zum Einsatz. Flyer, Ratgeberbroschüren und Informationsvideos wurden an Betroffene, Angehörige und Interessierte verteilt und in zahlreichen öffentlichen Veranstaltungen wurde auf das Thema aufmerksam gemacht. Ein Medienguide zur Berichterstattung über Suizid wurde erstellt, der auf den «Werther-Effekt» hinweist und für eine moderate Berichterstattung plädiert, um Nachahmungssuizide zu verhindern

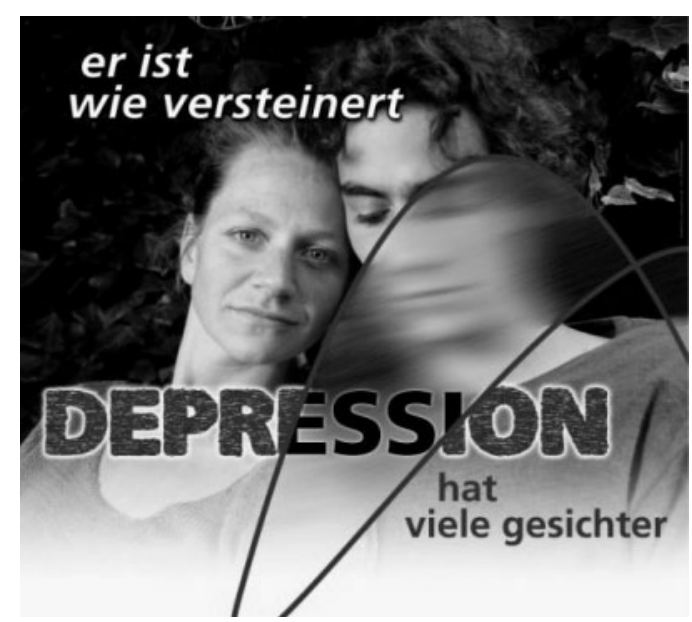

Abb. 2.

Eines von sechs Plakatmotiven, die bei der Kampagne in Nürnberg zum Einsatz kamen.

[Ziegler und Hegerl, 2002]. Unterstützt wurde die Öffentlichkeitsarbeit durch Bundesministerin Renate Schmidt und Staatsminister Dr. Günther Beckstein, die als Bürger Nürnbergs die Schirmherrschaft übernommen hatten.

3. Kooperation mit Multiplikatoren: Während der Kampagne wurden in rund 100 Fortbildungen Lehrer, Berater, Pfarrer, Altenpflegekräfte, Heilpraktiker, Polizisten und Apotheker zum Thema «Depression» und «Suizidalität» weiterqualifiziert. 4. Angebote für Betroffene und Angehörige: Hier ist zum einen die Einrichtung einer spezielle Telefon-Hotline für Patienten nach Suizidversuch zu nennen [Morgan et al., 1993]. Auf diese Art sollte im Falle einer Krise ein schneller und unbürokratischer Zugang zu einer fachärztlichen Behandlung hergestellt werden. Zum anderen wurden vor allem Selbsthilfeaktivitäten gefördert. Mit Hilfe der Koordination des «Bündnisses gegen Depression» ist es gelungen, zahlreiche neue Selbsthilfegruppen ins Leben zu rufen.

Die Frage war nun, ob sich durch diese Aktivitäten eine Reduktion der Suizidalität erzielen ließ. Prospektives primäres Erfolgskriterium war dabei die Veränderung der Häufigkeit suizidaler Handlungen (Suizide + Suizidversuche) während des Interventionszeitraumes (Januar 2001 bis Dezember 2002) gegenüber dem Vergleichsjahr 2000 (Baseline) und der Kontrollregion Würzburg. Im Vergleich zur Baseline und zur Kontrollregion konnte in der Tat in Nürnberg ein statistisch signifikanter und klinisch hochrelevanter Rückgang suizidaler Handlungen um $24 \%$ erreicht werden. Die Suizidversuche gingen dabei in Nürnberg um insgesamt 26\% zurück (Abb. 3), wobei der Rückgang für Suizidversuche mit «harten» Methoden wie Erhängen, Sprung aus großer Höhe, Überfahren, Ertrinken deutlicher war als für «weichere» Methoden wie Vergiftungen. Die Reduktion der Suizide betrug durchschnittlich $18 \%$, unterschied sich aber wegen der hohen jährlichen Fluktuationen nicht signifikant zur Kontrollregion und zur Baseline.

Der deutliche Rückgang suizidaler Handlungen während der 


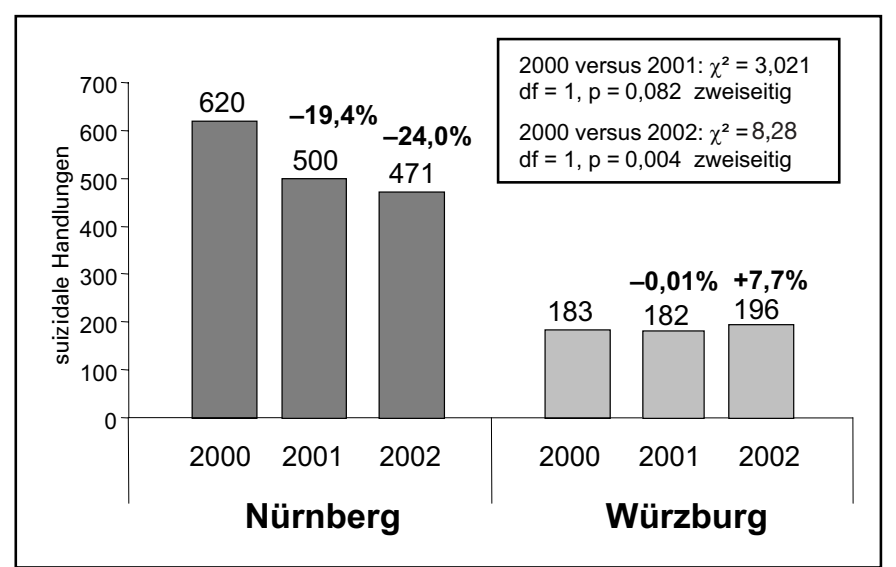

Abb. 3. Rückgang suizidaler Handlungen in Nürnberg.

Aktivitäten des Nürnberger Bündnisses gegen Depression sowohl gegenüber einem Vergleichsjahr als auch gegenüber einer Kontrollregion liefert starke Evidenz dafür, dass eine Vier-Ebenen-Intervention zur Optimierung der Versorgung depressiver Patienten suizidpräventiv wirksam ist. Damit steht erstmals eine sorgfältig evaluierte und evidenzbasierte Strategie zur Prävention suizidaler Handlungen zur Verfügung. Hierdurch wurde das Interesse vieler anderer Regionen in Deutschland und Europa geweckt. Die für Nürnberg erstellten Materialien und Konzepte können ohne viel Aufwand für andere Regionen adaptiert werden. Dadurch wird es für interessierte Regionen möglich, lokale Kampagnen mit geringem finanziellem Aufwand umzusetzen. Dieser Prozess der Ausdehnung der Nürnberger Kampagne auf andere Regionen wird im Rahmen der dritten Förderperiode des Kompetenznetzes «Depression, Suizidalität» (2005-2008) und des gemeinnützigen Vereins «Bündnis gegen Depression e.V.» gefördert. Die Partnerregionen erhalten vom Verein umfangreiche Unterstützung (unter anderem Logo, Plakatmotive, Informationsflyer, Kinospot, Videos, Fortbildungspakete für Hausärzte, Altenpfleger, Pfarrer und andere Berufsgruppen, gemeinsamen Bündnishomepage (www.buendnis-depression.de). Ein überregionaler Koordinator steht für Fragen der Evaluation und Kampagnendurchführung sowie zur Durchführung von regional angebotenen «Train-the-Trainer»-Seminaren zur Verfügung. Bis Ende 2004 haben zahlreiche Regionen eigene regionale Initiativen gegründet (Abb. 4), andere Regionen sind in der Vorbereitung entsprechender Aktivitäten.

Anknüpfend an das erfolgreiche Konzept des «Bündnisses gegen Depression» in Deutschland wurde im Frühjahr 2004 das «Europäische Bündnis gegen Depression (European Alliance Against Depression, EAAD)» ins Leben gerufen. Mit Unterstützung durch die Europäische Kommission (Generaldirektorat Verbraucherschutz und Öffentliche Gesundheit) haben sich 18 Partner aus 16 europäischen Ländern zusammengeschlossen, um zunächst in ausgewählten Regionen ähnliche Aktionsprogramme zu etablieren, wie dies in vielen

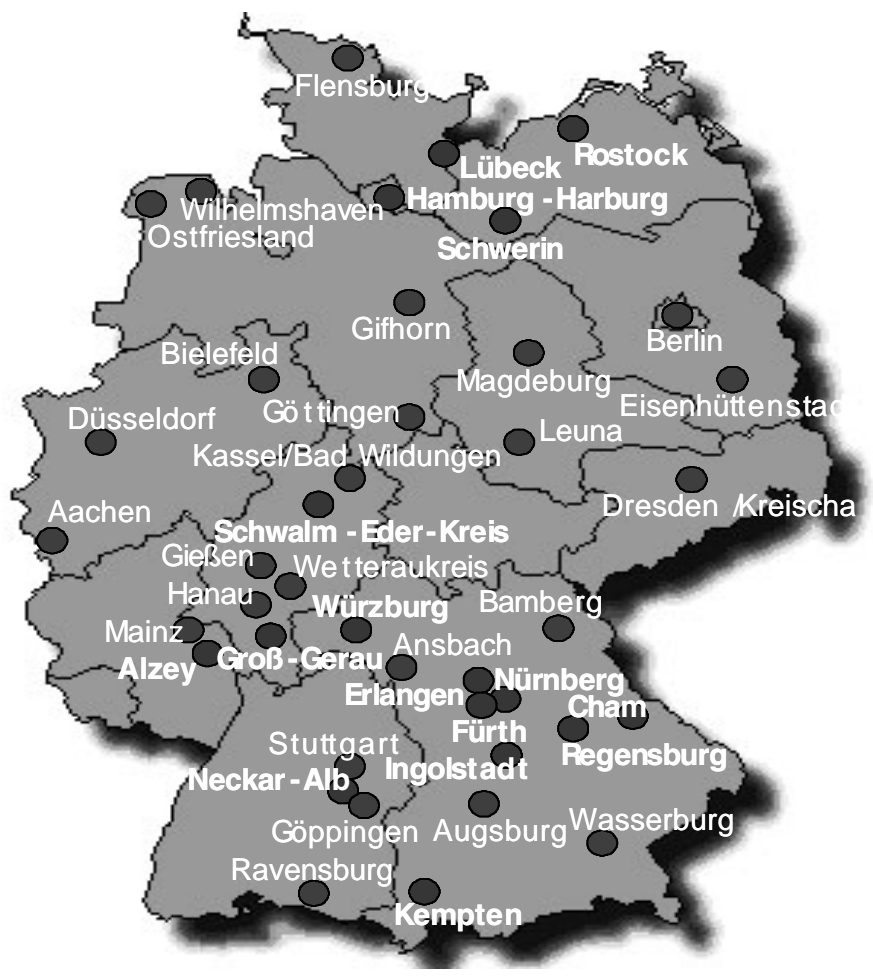

Abb. 4. Fett gedruckt sind die gegenwärtigen Partner des Bündnisses gegen Depression e.V. In normaler Schrift stehen Städte und Regionen, die einen Beitritt zum Bündnis erwägen oder konkret vorbereiten.

Regionen Deutschlands im Rahmen des «Bündnisses gegen Depression» bereits geschehen oder geplant ist. Nähere Informationen sind der Website www.eaad.net zu entnehmen.

\section{Effekte von Antidepressiva auf die Suizidalität}

Bei Erwachsenen gibt es sehr gute Belege dafür, dass Antidepressiva die Depressivität und damit auch suizidale Gedanken und Impulse reduzieren. Noch bedeutsamer ist, dass durch Antidepressiva das Rezidivrisiko gegenüber Placebo um $60-80 \%$ gesenkt wird und damit erneute Krankheitsphasen mit erhöhter Suizidalität vermieden werden können. Intensiver diskutiert wird in den letzten Monaten jedoch auch die Frage, ob Antidepressiva und insbesondere SSRIs (selektive Serotonin-Wiederaufnahmehemmer) zu einer Zunahme der Suizidalität bei einzelnen Patienten führen können. Ausgelöst wurde diese Diskussion durch kritische Stellungnahmen von britischen und amerikanischen Arzneimittelbehörden zur Antidepressivabehandlung von depressiven Kindern und Jugendlichen. Diese kritischen Äußerungen basieren vor allem darauf, dass die Wirksamkeit von Antidepressiva bei Kindern und Jugendlichen weniger untersucht ist und in mehreren Studien die Wirksamkeit gegenüber Placebo nicht belegt werden konnte [vgl. Gunnell und Ashby, 2004]. Hinzu kam, dass unter der Behandlung mit SSRIs und anderen Anti- 
depressiva bei Kindern und Jugendlichen ein häufigeres Auftreten suizidaler Impulse als unter Placebo beobachtet wurde [Jureidini et al., 2004]. Auch wenn es im Rahmen dieser Studie nicht zu Suiziden kam, so sind diese Beobachtungen ernst zu nehmen und waren vor dem Hintergrund der ungenügenden Wirksamkeitsbelege Anlass, vor einem breiteren Einsatz von Antidepressiva bei Kindern und Jugendlichen zu warnen. In der sich entwickelnden Diskussion wurde das Thema dann auch auf die Gruppe der Erwachsenen ausgeweitet, was zu Verunsicherung zahlreicher Patienten und Ärzten geführt hat [vgl. Hegerl, 2004].

Dass gerade zu Beginn einer Behandlung mit Antidepressiva die Suizidalität mit besonderer Sorgfalt beachtet werden muss, ist Lehrbuchwissen [vgl. auch Pfennig et al., 2005]. Durch initial auftretende Nebenwirkungen, die im Rahmen schwerer Depressionen als weiterer Beleg für die Hoffnungslosigkeit der Situation interpretiert werden, durch initiale Agitiertheit und durch Rückgang der Antriebshemmung bei Weiterbestehen der Hoffnungslosigkeit und der suizidalen Impulse kann gerade in den ersten Wochen einer Behandlung die Suizidalität zunehmen. Dies gilt übrigens auch für Psychotherapie und andere Behandlungsformen. Dies wird kaum bestritten werden. Die zentrale Frage im Rahmen der Diskussion über Antidepressiva und Suizidalität ist deshalb, wie groß das Risiko unter einer antidepressiven Behandlung ist, und insbesondere, ob das Risiko für einige Substanzen wie SSRIs höher als für andere Antidepressiva ist.

Eine Studie von Isacsson et al. [2004] erlaubt die Abschätzung der Gefahr, die von SSRIs hinsichtlich Suizidalität ausgeht. Über acht Jahre hinweg wurden in Schweden bei allen Menschen, die sich das Leben genommen hatten, systematisch toxikologische Untersuchungen mit Bestimmung von Medikamenten durchgeführt. In der Altersgruppe bis 14 Jahre kam es zu 52 Suiziden. Bei 7 der Suizidopfer konnten Antidepressiva nachgewiesen werden, keines der Antidepressiva war jedoch ein SSRI. Auch in der Gesamtgruppe der insgesamt 14857 Suizidopfer wurden SSRIs seltener, als zu erwarten gewesen wäre, gefunden. Dies bedeutet, dass über 9 Jahre hinweg in ganz Schweden kein Kind unter 15 Jahren durch ein SSRI in den Tod getrieben worden ist. Diese Daten sind mit einer deutlichen Suizid induzierenden Wirkung der SSRIs kaum kompatibel. Nicht ausgeschlossen werden kann durch diese Untersuchung, dass Antidepressiva das Suizidversuchsrisiko gegenüber Placebo erhöhen, wie durch die Reanalyse von Studien bei Kindern und Jugendlichen und einem neueren systematischen Review randomisierter kontrollierter Studien nahe gelegt wird [Fergusson et al., 2005].

Von Interesse ist in diesem Zusammenhang auch die Studie von Khan et al. [2003], in der rückwirkend die Studiendaten der Amerikanischen Zulassungsbehörde für Medikamente (FDA) ausgewertet wurden. In diesen Studien mit zirka 50000 Patienten unterschied sich das Suizidrisiko unter SSRI nicht von dem unter anderen Antidepressiva inklusive der älteren tri- und tetrazyklischen Antidepressiva. Das Suizidrisiko war numerisch sogar niedriger. Bemerkenswert an der Studie von Khan et al. [2003] ist zusätzlich, dass sich die Suizidrate unter Antidepressiva statistisch auch nicht von der unter Placebo unterschied und damit auch keinen Beleg für eine suizidpräventive Wirkung der Antidepressiva lieferte. Dies ist zum Teil dadurch zu erklären, dass sich in den hier untersuchten Akutstudien bei der bekannten Wirklatenz der Antidepressiva und der nur moderaten Placebo-Verum-Differenz in der antidepressiven Wirksamkeit Effekte auf das Suizidrisiko nur schwer zeigen können und zudem akute Suizidalität Ausschlussgrund gewesen ist. Weiter ist zu bedenken, dass auch in dieser Metaanalyse die Zahl der randomisierten Patienten nur ein Anteil dessen ist, was nötig wäre, um eine Verdoppelung oder Halbierung der Suizidraten gegenüber Placebo statistisch zu sichern. Diese beiden genannten Studien liefern immerhin Hinweise dafür, dass es keine größeren Unterschiede zwischen SSRIs und anderen Antidepressiva bezüglich einer möglichen Induktion von Suiziden gibt.

Für die Gruppe der Antidepressiva insgesamt gibt es eine Reihe indirekter Argumente, dass diese zu einer Reduktion des Suizidrisikos bei den damit behandelten Patienten führen. So wurde bei einer in Australien durchgeführten Studie von Hall et al. [2003] gefunden, dass in den Geschlechts- und Altersgruppen, in denen der stärkste Anstieg von Antidepressivaverschreibungen zu verzeichnen war, auch der Verlauf in den Suizidraten am günstigsten war. Ein ähnlicher $\mathrm{Zu}$ sammenhang wurde für Kinder und Jugendliche in den USA gefunden, wo die nach Postleitzahl getrennten Regionen mit der stärksten Zunahme der Antidepressivaverschreibung ebenfalls die stärkste positive Veränderung im Bereich der Suizidraten aufwiesen [Olfson et al., 2003].

In dieser gesamten Diskussion wird ein für die Praxis höchst relevanter Aspekt viel zu wenig diskutiert: die Bedeutung der Überdosierungssicherheit der Antidepressiva als Auswahlkriterium bei der Behandlung potentiell suizidaler Patienten [vgl. Pfennig et al., 2005]. Während die Einnahme einer Wochenration von trizyklischen Antidepressiva in suizidaler Absicht letale Folgen haben kann, werden exzessiv hohe Dosen von SSRIs meist ohne Spätfolgen überlebt. Eine Auswertung der Suizidversuche im Rahmen der Studie «Nürnberger Bündnis gegen Depression» ergab, dass Intoxikationen mit Psychopharmaka mit großem Abstand die am häufigsten gewählte Suizidversuchsmethode waren und dass $13 \%$ aller Suizidversuche mit Antidepressiva (ca. 60\% hiervon mit tri- und tetrazyklischen Antidepressiva) erfolgten. Auf Deutschland hochgerechnet ist mit 10000 Suizidversuchen mit Antidepressiva jährlich zu rechnen. Zahlreiche stationäre intensivmedizinische Behandlungen und Spätfolgen könnten vermieden werden, wenn bei suizidalen Patienten weniger trizyklische Antidepressiva und mehr Antidepressiva mit hoher Überdosierungssicherheit eingesetzt würden.

Insgesamt ergibt sich als Konsequenz für die klinische Praxis, dass entsprechend bekanntem Lehrbuchwissen gerade zu Beginn einer Behandlung und vielleicht verstärkt bei Einsatz 
aktivierender Antidepressiva auf eine mögliche Zunahme der Suizidalität $\mathrm{zu}$ achten ist und gegebenenfalls eine engermaschige Betreuung oder zusätzlich sedierende Begleitmedikation notwendig sind. Auch sollten die Patienten auf die Möglichkeit einer initialen Zunahme der Suizidalität unter einer antidepressiven Behandlung hingewiesen werden.

\section{Dank}

Diese Arbeit entstand im Rahmen des Kompetenznetzes Depression, Suizidalität und wurde vom Bundesministerium für Bildung und Forschung (BMBF) unterstützt.

\section{Literatur}

Althaus D, Stefanek J, Hasford J, Hegerl U: Wissensstand und Einstellungen der Allgemeinbevölkerung zu Symptomen, Ursachen und Behandlungsmöglichkeiten depressiver Erkrankungen. Nervenarzt 2002;73 659-664.

Angst F, Stassen HH, Clayton PJ, Angst J: Mortality of patients with mood disorders: Follow-up over 34-38 years. J Affect Disord 2002;68:167-181

Angst J: Consensus discussion. J Clin Psychiatry 1999; 60:113-116.

Black DW, Winokur G, Mohandoss E, Woolson RF, Nasrallah A: Does treatment influence mortality in depressives? A follow-up of 1,076 patients with major affective disorders. Ann Clin Psychiatry 1989;1:165173.

Blair-West GW, Cantor CH, Mellsop GW, EyesonAnnan ML: Lifetime suicide risk in major depression: Sex and age determinants. J Affect Disord 1999;55: 171-178.

Brent DA, Perper J, Moritz G, Allmann C, Friend A, Roth C, Schweers J, Balach L, Baugher M: Psychiatric risk factors for adolescent suicide: A case-control study. J Am Acad Child Adolesc Psychiatry 1993;32: 521-529.

Cheng AT: Mental illness and suicide - a case-control study in East Taiwan. Arch Gen Psychiatry 1995;52: 594-603.

Clayton PJ: Epidemiologic and risk factors for suicide in Grinspoon L (ed): Psychiatry Update. Washington, DC, American Psychiatric Press, 1983, pp 428-434.

Fergusson D, Doucette S, Cranley Glass K, Shapiro S, Healy D, Hebert P, Hutton B: Association between suicide attempts and selective serotonin reuptake inhibitors: Systematic review of randomised controlled trials. BMJ 2005;330:396.
Gunnell D, Ashby D: Antidepressants and suicide: What is the balance of benefit and harm? BMJ 2004; 329:34-38.

Guze SB, Robins E: Suicide and primari affective disorder. Br J Psychiatry 1970;117:437-438.

Hall WD, Mant A, Mitchell PB, Rendle VA, Hickie IB, McManus P: Association between antidepressant prescribing and suicide in Australia, 1991-2000: Trend analysis. BMJ 2003;326:1008.

Hegerl U: Antidepressiva und Suizidalität. Verhaltenstherapie 2004;14:218-219.

Hegerl U, Althaus D, Stefanek J: Public attitudes towards treatment of depression: Effects of an information campaign. Pharmacopsychiatry 2003;36:288-291. Henkel V, Mergl R, Kohnen R, Maier W, Moeller H, Hegerl U: Identifying depression in primary care: A comparison of different methods. BMJ 2003;226:200221.

Inskip HM, Harris EC, Barraclough B: Lifetime risk of suicide for affective disorder, alcoholism and schizophrenia. Br J Psychiatry 1998;172:35-37.

Isacsson G, Holmgren P, Ahlner J: Do SSRIs induce suicide? A controlled study of Swedish suicides 1992-2000. Presented at the 17th ECNP 2004 in Stockholm.

Jureidini JN, Doecke CJ, Mansfield PR, Haby MM, Menkes DB, Tonkin AL: Efficacy and safety of antidepressants for children and adolescents. BMJ 2004; 328:879-883

Khan A, Khan S, Kolts R, Brown WA: Suicide rates in clinical trials of SSRIs, other antidepressants, and placebo: Analysis of FDA reports. Am J Psychiatry 2003;160:790-792.
Lönnqvist J: Psychiatric aspects of suicidal behaviour: Depression; in Hawton K, van Heeringen K (eds): The International Handbook of Suicide and Attempted Suicide. Chichester, John Wiley \& Sons, 2000 Morgan HG, Jones EM, Owen JH: Secondary prevention of non-fatal deliberate self-harm. The green card study. Br J Psychiatry 1993;163:111-112.

Mortensen PB, Agerbo E, Erikson T, Qin P, Westergaard-Nielsen N: Psychiatric illness and risk factors for suicide in Denmark. Lancet 2000;355:9-12.

Olfson M, Shaffer D, Marcus SC, Greenberg T: Relationship between antidepressant medication and suicide in adolescents. Arch Gen Psychiatry 2003;60:978982

Pfennig A, Berghöfer A, Bauer M: Medikamentöse Behandlung der Suizidalität. Verhaltenstherapie 2005 DOI: $10.1159 / 000083490$

Simon GE, Von Korff M, Piccinelli M, Fullerton C, Ormel J: An international study of the relation between somatic symptoms and depression. N Engl J Med 1999;341:1329-1335.

Tsuang MT, Woolson RF: Excess mortalità in schizophrenia and affective disorders. - Do suicides and accidental deaths solely account for this excess? Arch Gen Psychiatry 1978:35:1181-1185.

WHO (ed): World Health Organisation Info Package: Mastering Depression in Primary Care. Frederiksborg, WHO, 1998.

Wittchen H-U, Müller N, Schmidtkunz B, Winter S, Pfister H: Erscheinungsformen, Häufigkeit und Versorgung von Depressionen. Fortschr Med 2000, 118(S1):4-10.

Ziegler W, Hegerl U: Der Werthereffekt: Bedeutung, Mechanismen und Konsequenzen. Nervenarzt 2002;73: 41-49. 